\title{
Real-Time Measurements in Optical Metrology
}

\author{
H.J. TIZIANI \\ Institut für Technische Optik \\ Universität Stuttgart \\ D-7000 Stuttgart 80
}

\section{Introduction}

Optical methods for contactless testing are widely used. Mostly responsible for it is the introduction of the laser in 1960. Numerous applications such as contactless distance, velocity, shape deformation, displacement and vibration analysis use the laser as a light source. Furthermore, different acousto-optical and electrooptical modulation-and beam deflection devices were introduced $[1,2]$. Acousto-optical devices are widely used as modulators and deflectors; they are also used in fast printers. Electrooptical modulation systems and beam deflectors are based on the Pockels-effect, acousto optical on the Bragg-effect. For the Pockels Readout Optical Modulator (PROM) the electrical field was applied parallel to the optical axis.

The PROM is a real-time, recyclable, spatial light modulator constructed from a wafer of bismuth silicon oxide. The variation of the refractive index of the crystal is based on both, photoconductivity and the linear electrooptic Pockels-effect.

The electrooptic modulator works as follows: During readout, the incident linearly polarized light becomes elliptically polarized via the linear electrooptic effect of the crystal, with the degree of ellipticity dependent on the spatially varying voltage across the crystal leading to the appropriate refractive index change. When the light is passed through an analyser, the output intensity depends on the square of the sine of the voltage of the crystal. After readout the image is erased with.a flash light of the appropriate wavelength (blue). Further applications of the PROM were in the field of real-time recyclable image storage devices in coherent and incoherent optical processing systems [3]. Real-time correlationand image detail enhancement systems were studied. In addition, hybrid field-effect liquid crystal light valves for real-time coherent optical data processing were investigated. The light valve is basically a high resolution optical to - optical image transducer capable of accepting a low intensity (white or green) input light image and converting it, in real-time into an output image with light from another source [4]. The device can be designed so that the input and output light beams are completely separated and noninteractive.

For holographic storage techniques for write/read applications, high recording density, sensitivity and fast erasure are required. Volume phase holography techniques for the storage of graphic or binary information in holographic form throughout the entire volume of a thik storage medium are promising. 
Ferroelectric storage materials offer good storage capacity, high readout efficiency, high resolution and reversibility. The most widely used material is $\mathrm{Fe}$ doped $\mathrm{Li} \mathrm{Nb} \mathrm{O}_{3}[5]$. Three different mechanisms for charge migration were identifiéd namely diffusion, drift in an electric field and transport due to an internal photovoltaic effect.

The application of holography in optical metrology such as holographic interferometry for contactless optical displacement, deformation and vibration analysis is well known. Storage materials are mainly photographic materials based on silver halide; photoresists, dichromated gelatine, photochromic-, thermoplastic materials and electrooptical crystals are possible alternatives. Photothermoplastic material is already used for holography and holographic interferometry. An example of speckle pattern recording on thermoplastic material is shown in fig. 10 .

The most promising electrooptical materials are bismuth silicon oxide $\mathrm{Bi}_{12} \mathrm{SiO}_{20}$ (BSO) and bismuth germanium oxide $\mathrm{Bi}_{2} \mathrm{GeO}_{20}$ (BGO). The writing energy for a diffraction efficiency of 1 is is $0,9 \mathrm{~mJ} / \mathrm{cm}^{2}$ for BSO and $1.25 \mathrm{~mJ} / \mathrm{cm}^{2}$ for BGO as compared with $30 \mathrm{~mJ} / \mathrm{cm}^{2}$ for $\mathrm{Li}$ $\mathrm{NbO}_{3}$ (1ithium niobate). The sensitivity of BSO is comparable with the fine grain holographic emulsion Kodak $649 \mathrm{~F}$ but less sensitive than thermoplastic material. We will be mostly concerned with the BSO crystals for contactless optical measurements. The applications with the BSO are nearly real-time applications.

\section{Real - time holography and speckle application with BSO cry- stals}

Holographic interferometry and speckle applications for deformation, displacement and vibration analysis are widely known. Holography and holographic interferometry are well understood. The correlation between the fringe pattern obtained and the deformation is not always easily obtainable. The applications of the technique will be even more attractive to the engineer when it can be used in real-time.

For real-time holographic and speckle application, the BSO, a cubic crystal, is usually biased with a transverse electric field $E_{0}$ in the 110 crystal - lographic direction as shown in fig. 1 . When the crystal is illuminated with spatially structured illumination as a result of a hologram or a speckle pattern in the $T 10$ direction, a space charge field is built up. The physical mechanisms for holography and speckle pattern recording and erasure are drift and trapping of photoelectrons under illumination by a light pattern. In the following applications the crystal is used in the transverse electrooptic configuration. The photoinduced space charge field changes the refractive index of the crystal via the linear electrooptic effect, leading to a refractive index variation in the crystal volume. Flooding with uniform illumination leads to the erasure of the stored information by space charge relaxation. Consequently, reading out with the recording wavelength is destructive. The information desired can be stored with TV-techniques, the fringe pattern from holographic interferometry or speckle applications can be analysed electronically. The BSO's used are usually sensitive in the bluegreen spectral region. A red sensitive crystal was also used in our laboratory. The transverse electric field applied was $6 \mathrm{kV} / \mathrm{cm}$ and the dark storage time of the information was found to be. $30 \mathrm{~h}$. 


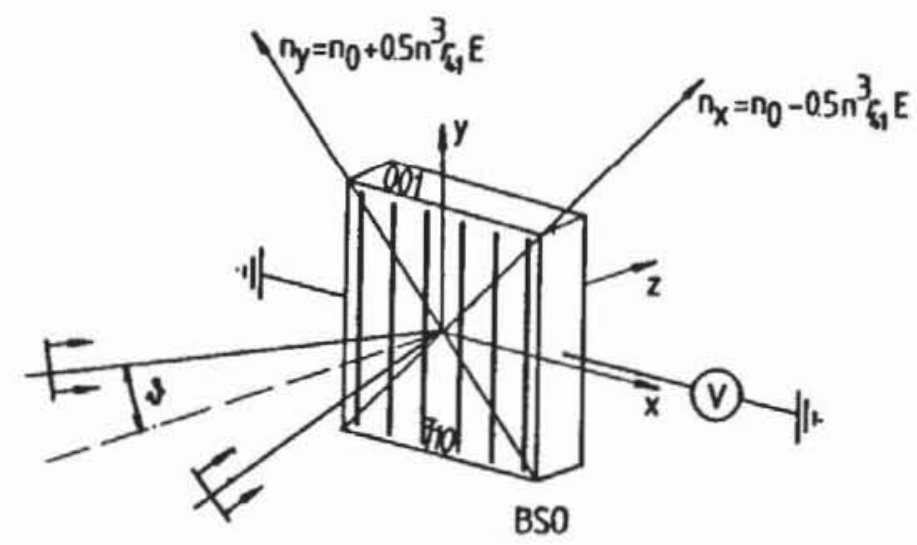

Fig. 1 Application of the BSO crystal with a transverse electric field

\subsection{Real - time holographic interferometry}

A typical arrangement for holographic recording and reconstruction in real-time is shown in fig. 2. The object is illuminated with coherent light. The object-and coherent reference waves are stored in the crystal. A mirror reflects the reference wave appropriately, leading to the reconstruction of the object wave; the Bragg condition has to be satisfied. The reconstructed phase conjugate object wave is seen via a beam splitter. Since the reconstructed wave is not only reflected but is also phase conjugated, disturbances introduced in the recording stage are compensated by the reconstruction through the disturbing medium [7].

For real-time holographic interferometry in the case of double exposure, the hologram of the object is stored in the crystal. The second exposure occurs after the deformation of the object has taken place.

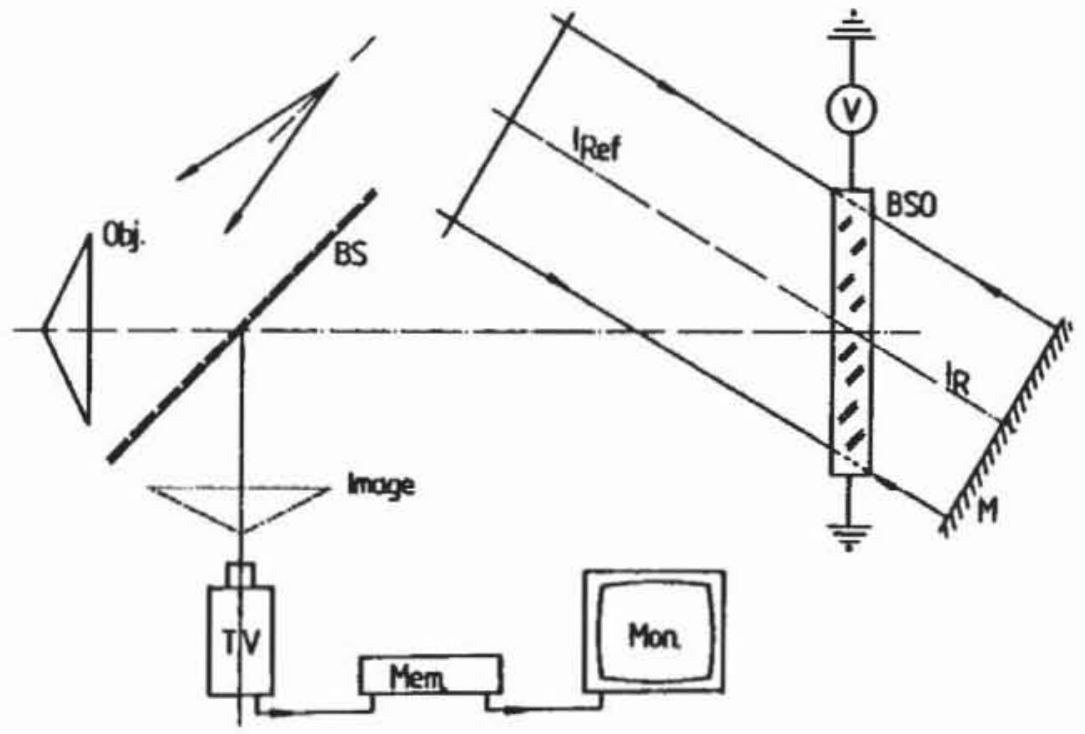

Fig. 2 Arrangement for real-time holographic recording and display with a BSO crystal 


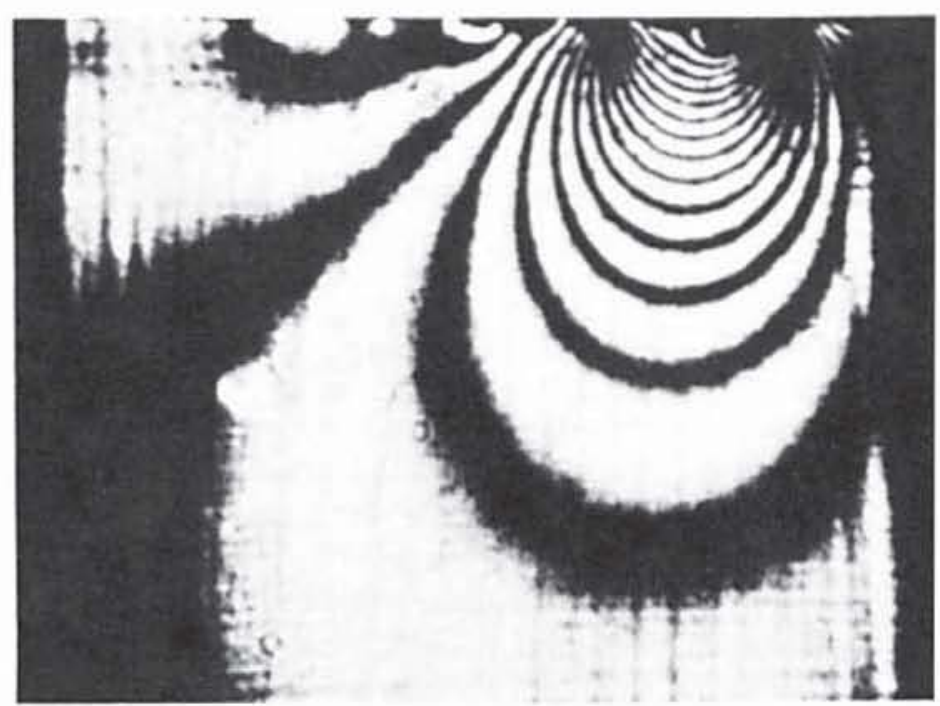

Fig. 3 Example of a holographic double exposure for deformation measurement

The original and the deformed wavefronts are then reconstructed leading to interference fringes related to the deformation. Fringes occur when the second exposure takes place. Fig. 3 shows the result of a deformed matallic plate. By contrast, fig. 4 shows the result of a vibrating membrane recorded in the time average with an exposure time of $1 \mathrm{sec}$. The frequency of oscillation was $300 \mathrm{~Hz}$ for fig. 4a) and $1.500 \mathrm{~Hz}$. for fig. $4 \mathrm{~b})$

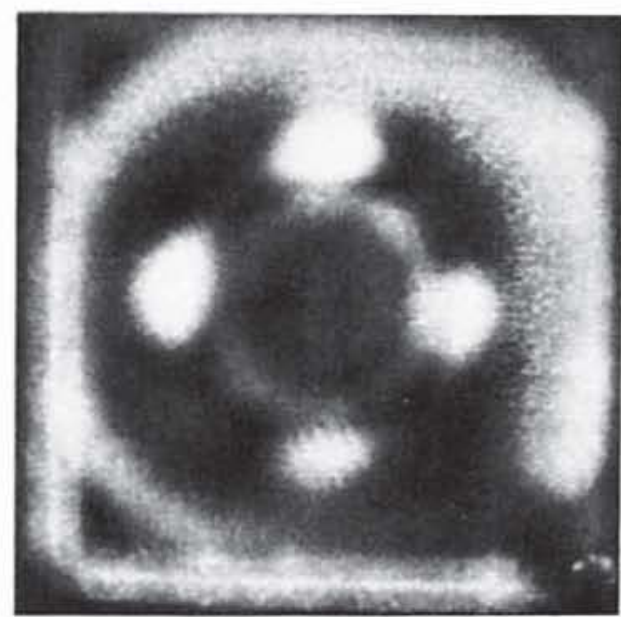

a)

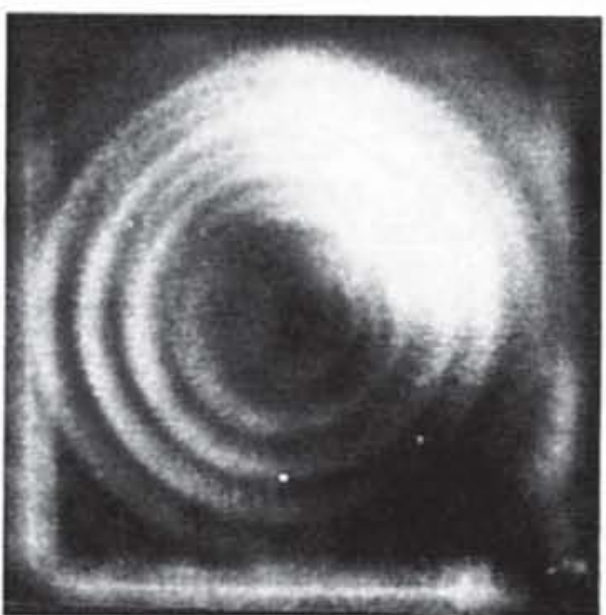

b)

Fig. 4 Fringes of a vibrating membrane recorded in the time average. The oscillating frequencies were for
a) $300 \mathrm{~Hz}$
and for
b) $1.500 \mathrm{~Hz}$ 


\subsection{Real - time contour holography}

Contour holography can be a powerful tool for contactless measurements. Its application in real-time is even more useful for the engineer. The BSO crystal was found to be very appropriate for realtime contour holography [8]. An experimental arrangement for the two wavelength technique is shown in fig. 5 .

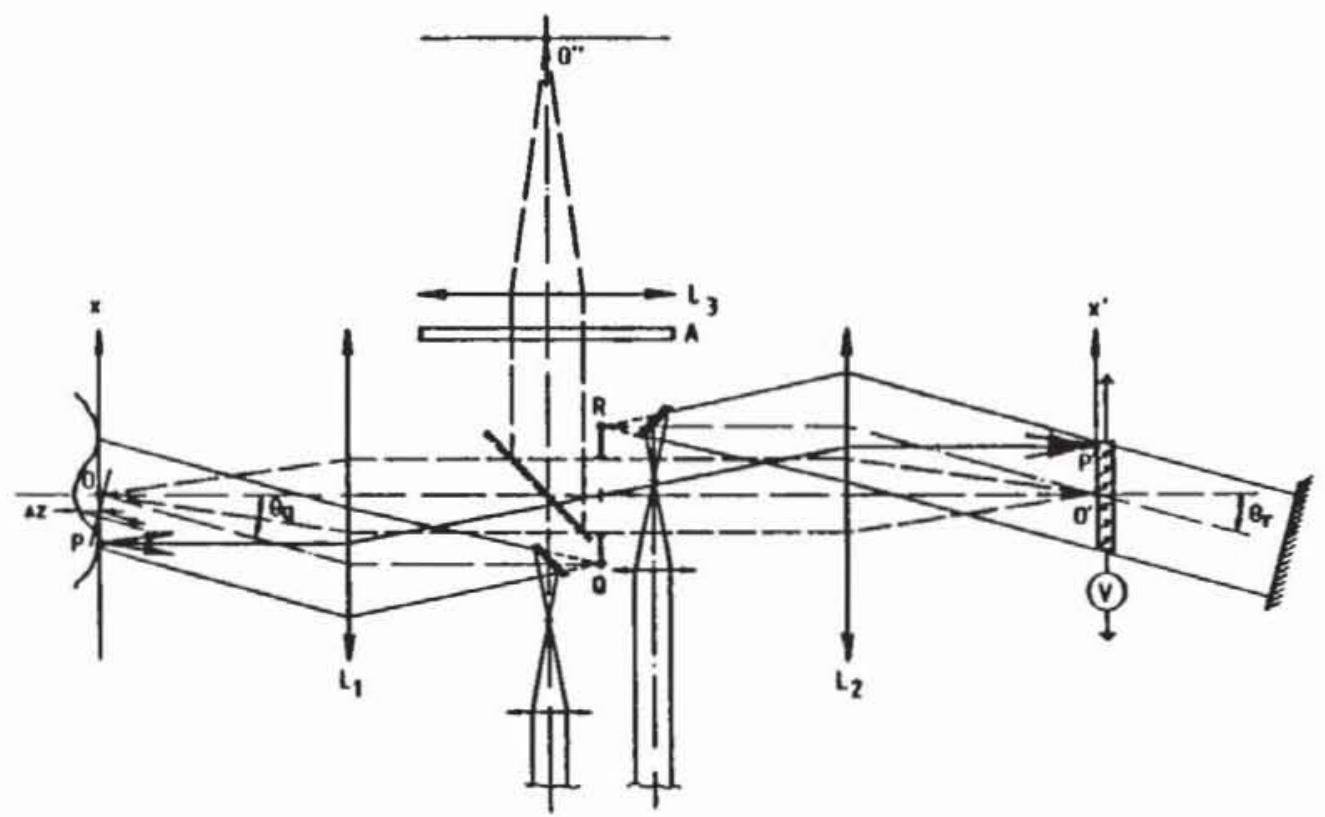

Fig. 5 Experimental arrangement for two wavelengths real-time holographic recording for contour line display

The object was illuminated simultaneously with two wavelength $\lambda_{1}$ and $\lambda_{2}$. The angle of the incident plane wave was $\theta_{\text {. The lenses }}$ $L_{1}$ and $L_{2}$ form the image of the object in the crystal with the reference wave of $\lambda$ and $\lambda_{2}$ superimposed. The reconstructing wave can be selected with the angle of the mirror, i.e. the reconstructing $\lambda$ satisfies the Bragg condition. Since the crystal is optically active, unwanted light can be blocked with the analyser $A$. In $O^{\prime \prime}$ the reconstruction occurs with contour lines. The separation of the contour lines is given by

$$
\Delta z=\frac{\lambda_{1} \lambda_{2}}{\left(1+\cos \theta_{q}\right)\left(\lambda_{1}-\lambda_{2}\right)}
$$

Fig. 6 shows a reconstructed image of a coin $6 a$ ) without and $6 \mathrm{~b}$ ) with contour lines. For this experiments two $\lambda$ 's of a Krypton laser were used. The spacing of the contour lines in fig. $6 \mathrm{~b}$ is $13,9 \mu \mathrm{m}$. other configuration for real-time contouring can be used. 


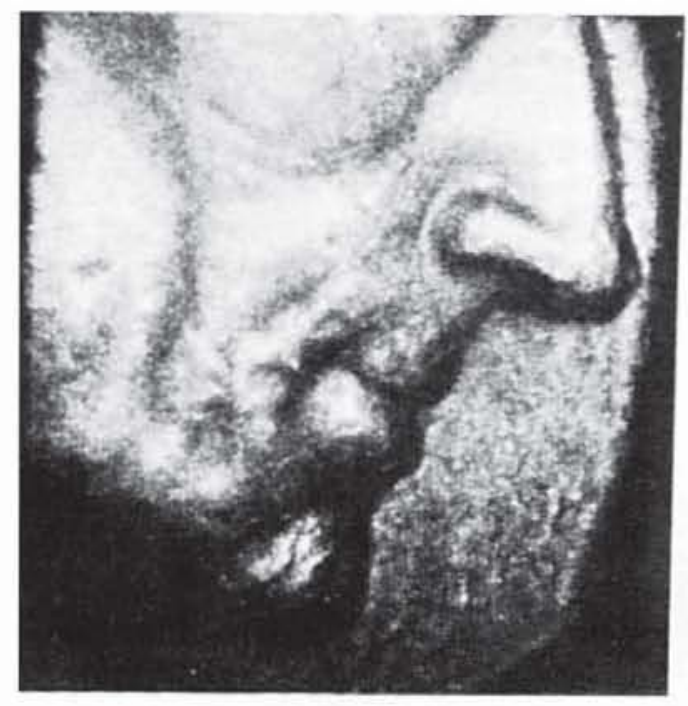

a

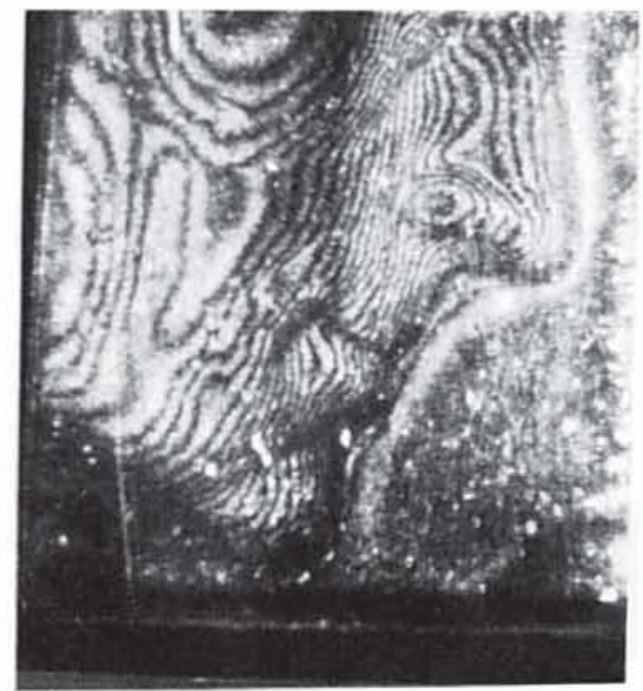

$\mathrm{b}$

Fiq. 6 Reconstructed real-time images of a coin
a) without contour lines
b) with contour lines with $\Delta z=13,9 \mu \mathrm{m}$

\section{Real - time speckle applications \\ 3.1 Real - time speckle applications with BSO crystals}

Real-time "speckle photography" by double exposure technique [9] or in the time average [10] was found attractive to the engineer. The experimental layout is shown in fig. 7. For the double exposure tech. nique, the speckle pattern is recorded before and after the deformation or displacement. The Yound's interference fringes are obtained in the Fraunhofer plane. No reference wave is required and the wavelength $\lambda_{2}$ of the wave displaying the Young's fringes can be different from the speckle pattern recording wavelength $\lambda_{1}$. The absorption ${ }_{1}$ of the BSO for $\lambda_{1}=514 \mathrm{~nm}$ is $2 \mathrm{~cm}^{-1}$ and for $\lambda_{2} \stackrel{1}{=} 633 \mathrm{~nm}$ is $0,28 \mathrm{~cm}^{-1}$, hence no storage device is needed for the analysis of Young's fringes.(to be analysed automatically with TV-techniques as indicated, For a harmonically oscillating object the speckle pattern can be recorded in the time average in a BSO crystal. Young's fringes obtained this way follow no longer a cosine square law but the square of the Besselfunction of order zero and first kind. The minima lead to the amplitude of oscillation [10].

In fig. 8 fringes of a double esposed speckle pattern with a displacement of $20 \mathrm{\mu m}$ between exposures are shown. In fig. $8 \mathrm{~b}$ ) fringes of a time average recording of the speckle pattern of a harmonically oscillating tuning fork, $20=18 \mu \mathrm{m}$, are shown. The fringes in fig. $8 \mathrm{~b}$ ) are no longer equidistant. A $1 \mathrm{~mW}$ He $\mathrm{Ne}$ laser was used to display the fringes. The frequency of oscillation was nearly $1 \mathrm{KHz}$; fringes could be seen $1 \mathrm{sec}$. after starting the exposure. 


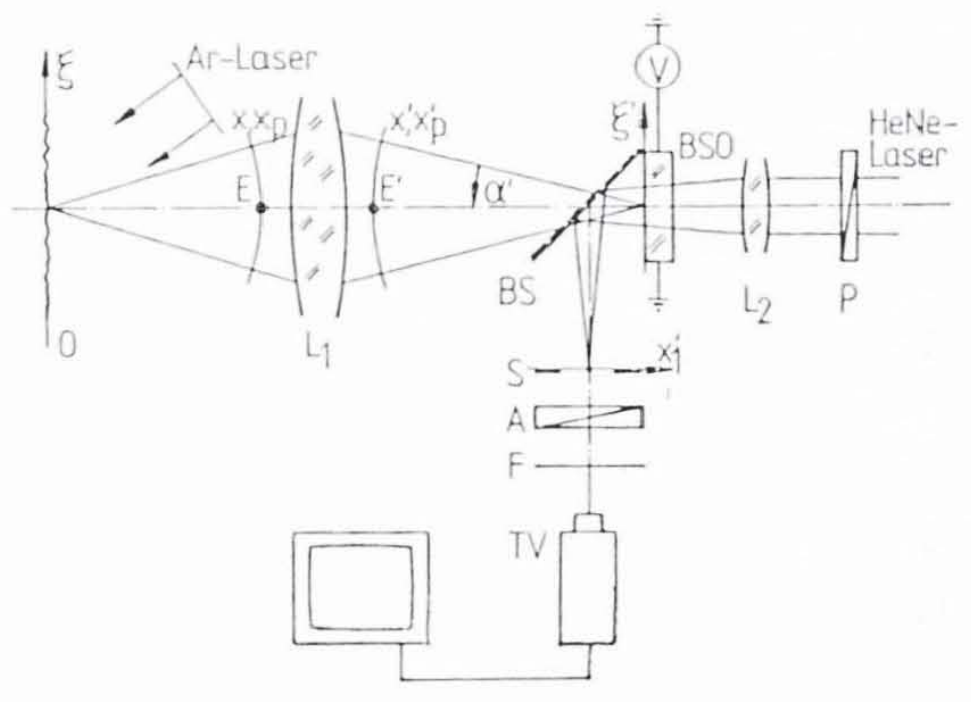

Fig. 7 Experimental arrangement for real-time speckle applications for deformation, tilt displacement- and vibration analysis

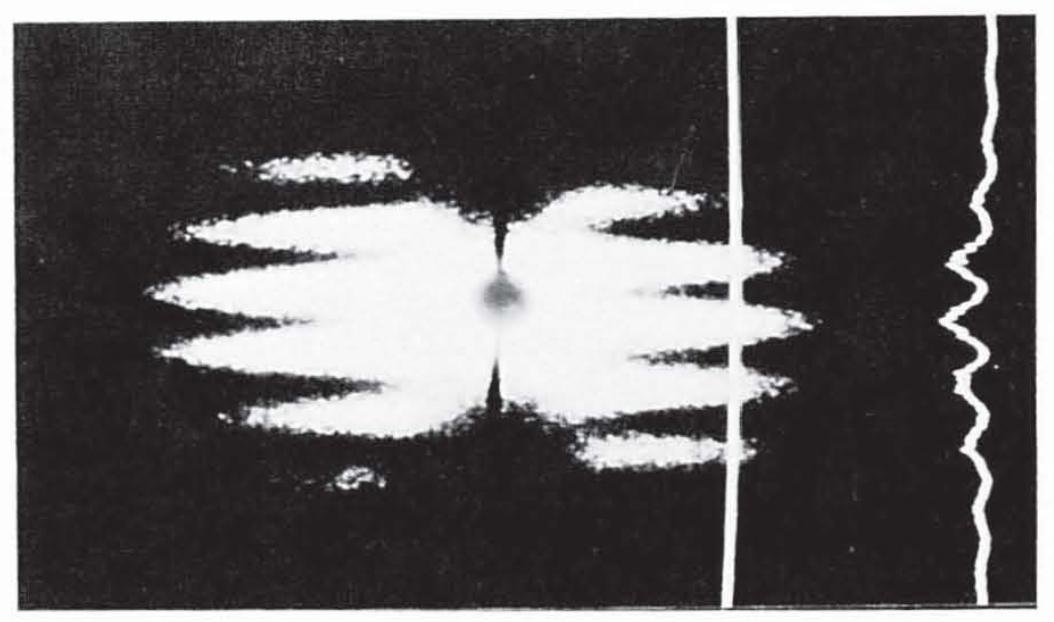

Fig. 8 a) Young's fringes of a double exposed speckle pattern with a displacement of 20 um between

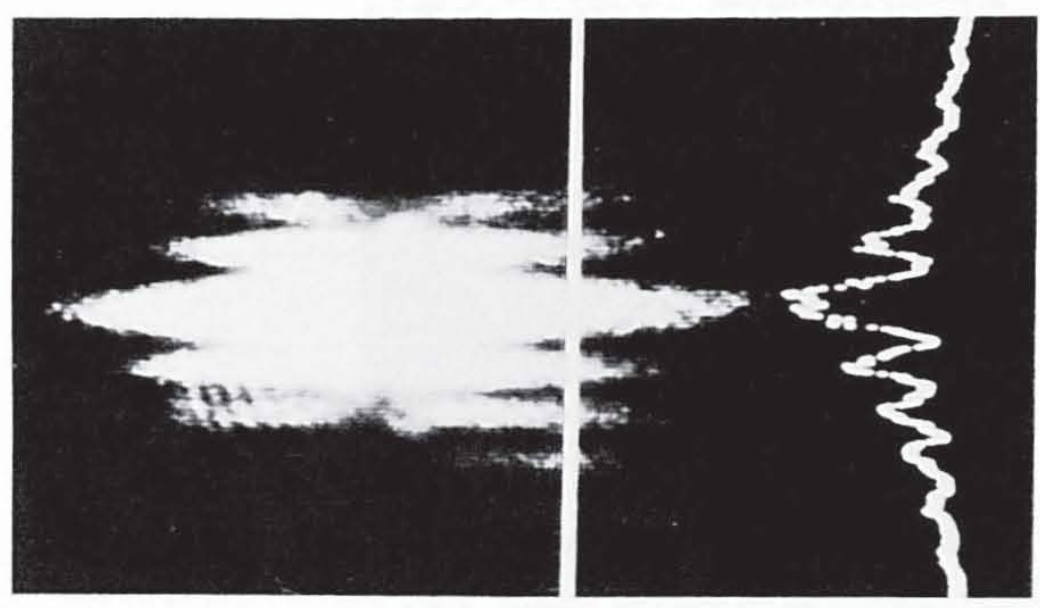

Fig. 8 b) time average recording of an oscillating tuning fork, $2 \mathrm{p}=18 \mathrm{~m}$ 
Thermoplastic photographic material, working in quasi real-time has been studied mainly as holographic recording material due to it's band pass properties. Schörner and Feiertag reported, at the conference, on instant holographic applications. The thermoplastic material can, however, also be used for quasi real-time speckle photography. The cycle time in our arrangement shown in fig. 9 was about $50 \mathrm{sec}$. In fig. 9 the image of the oscillating object is formed on the thermoplastic material. For displaying Young's fringes the thermoplastic material is illuminated with a narrow laser beam. The image of the fringes can be formed on a TV tube for the automatic analysis. Fig. 10 shows a fringe pattern of an oscillating tuning fork.

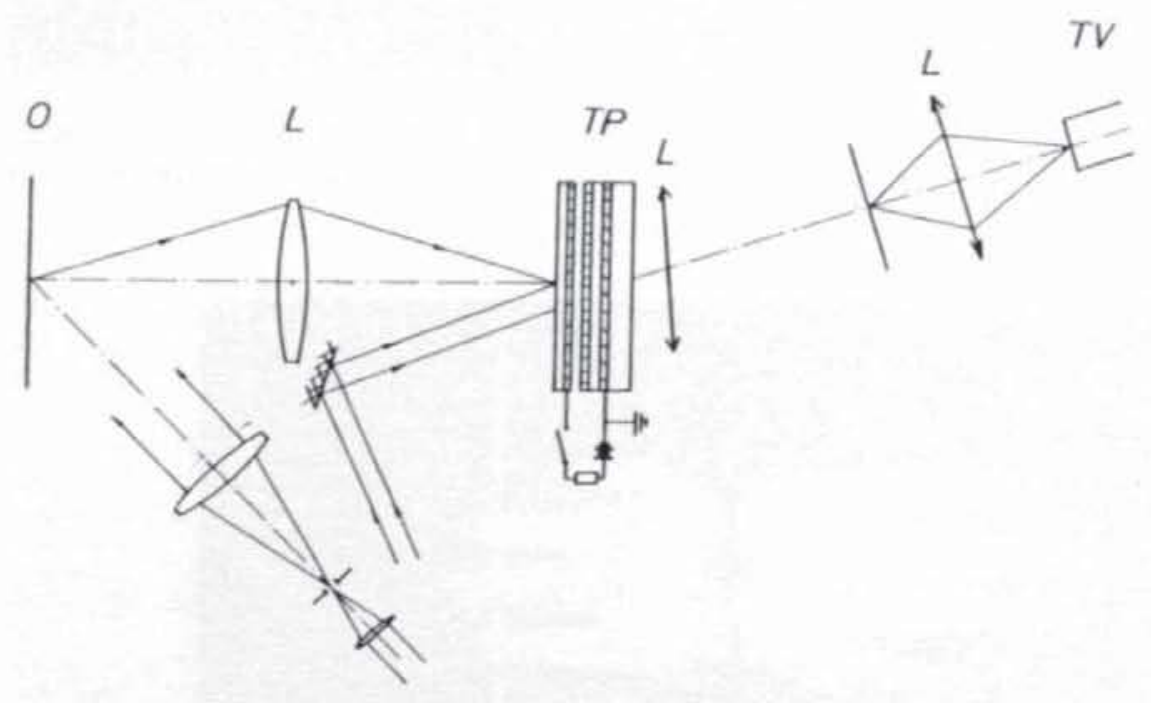

Fig. 9 Experimental arrangement for speckle pattern recording on thermoplastic material

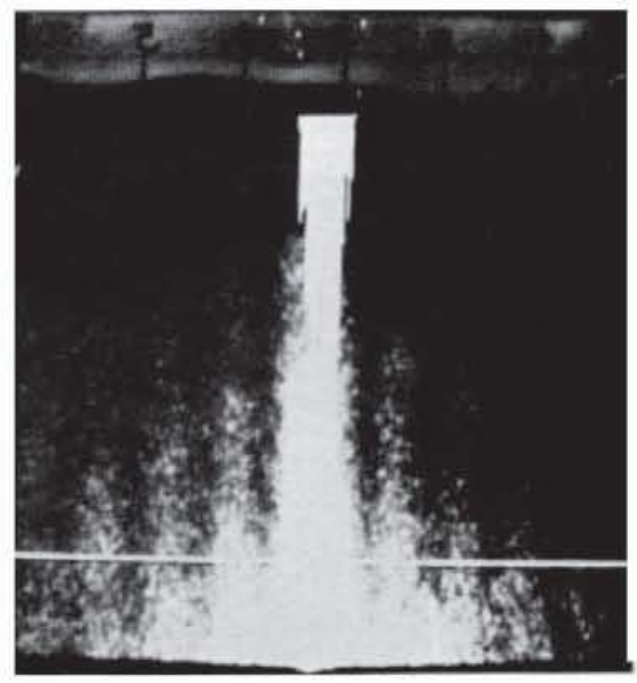

Fig. 10 Young's fringes obtained by recording an oscillating tuning fork, in the time average, on thermoplastic material 


\section{Real-time analysis with a Doppler shift vibrometer}

For some applications such as noise analysis in addition to the spatial distribution of the amplitude of the oscillating object, the time spectrum is of importance. For such an analysis a heterodyne technique can be used. The basic principle of such an arrangement is shown schematically in fig. 11 in a Twyman-Green arrangement. The incident wave of frequency $\omega_{1}$ is separated into two waves by the beam splitter following path (1) or $\theta$ before recombination. For the wave passing path 2 the frequency is shifted by the moving object of velocity $V(t)$ by $\frac{2 \pi}{\lambda} 2 \mathrm{~V}(t) \Delta t$. Furthermore, for convenience an additional frequency shift $\Delta \omega$ can be introduced by a Bragg cell in order to detect the siqn of the frequency shift i.e. the sign of $V(t)$. The interference pattern of the waves from path (1;) and $2:$ is for $\omega_{1}=w_{2}$

$I_{1}=a_{1}^{2}+a_{2}^{2}+\overline{2 a_{1} a_{2}} \cos \left(\phi_{1}-\phi_{2}\right)$

where $a_{1}, a_{2}$ and $\phi_{1}, \phi_{2}$ are the amplitude and phase respectively of the two interfering waves.

For $\omega_{1} \neq \omega_{2}$ the interference pattern to be analysed is $I_{2}=\overline{a_{1}^{2}+a_{2}^{2}} \pm 2 a_{1} a_{2} \cos \left(\Delta \omega t \pm \frac{2 \pi}{\lambda} 2 v(t) t+\left(\phi_{1}-\phi_{2}\right)\right)$

The analysis of the velocity component $V(t)$ perpendicular to the object surface is indicated schematically in fig. 11. With oblique illumination and a slightly modified arrangement, the real-time in plane velocity component can be found. For a harmonically oscillating object with the oscillation perpendicular to the object surface, the interference pattern to be analysed is

$$
I_{3}=a_{1}^{2}+a_{2}^{2}+2 a_{1} a_{2} \cos \left(\Delta \omega t \pm \frac{2 \pi}{\lambda} 2 \rho \cos \Omega t+\left(\phi_{1}-\phi_{2}\right)\right)
$$

from where the amplitude $\rho$ and frequencyshift $\Omega$ can be found.

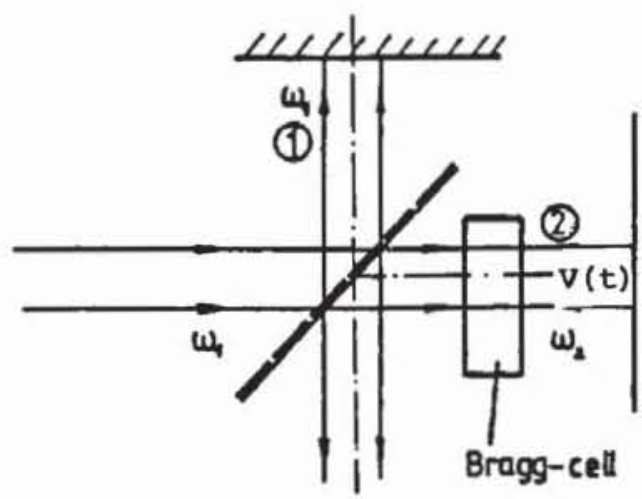

Vibrometer

Heterodyn detection

Fig. 11 Principle of a heterodyne technique for the velocity and vibration analysis 
Fig. 12 shows results of a vibration analysis obtained with different techniques. The amplitudes of oscillation of a tuning fork are plotted as function of the voltage applied. The full lines correspond to results from real-time speckle techniques and the broken from measurements with a Doppler shift vibrometer. The speckle techniques lead to the information of the spatial distribution of amplitude of oscillation. The Doppler shift vibrometer leads to the frequency and amplitude analysis at a given point of the object. Very often the two techniques are complementary.

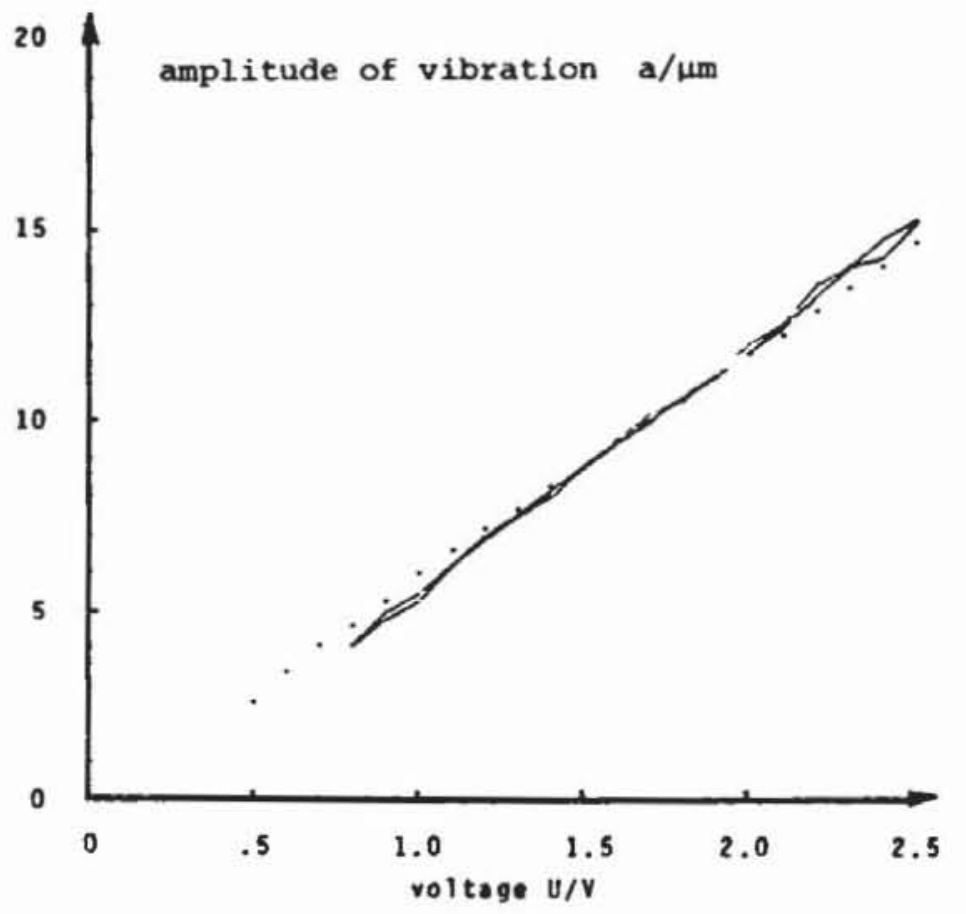

Fig. 12 Results of a Vibration analysis of an oscillating tuning fork with different techniques.

- _ - . - vibrometer with time average speckle technique in quasi real-time.

The amplitudes of oscillation are plotted as function of the voltage applied.

Results of a noise analyse at the side-wall of a rotating tyre with a simulated road contact by means of a toothed wheel are shown in fig. 13, for a noise analyse with a microphone (dashed line) and by analysing the mechanical vibrations with a Doppler shift vibrometer (full line). The results from contactless measurements with optical methods agree well with those of the noise analysis with microphones.

Holographic interferometry for quasi static objects is well understood by now. The correlation between the fringes of threedimensional deformation, displacement and vibration is often complicated without apriori information. The vibration analysis of rotating object requires usually the compensation of the rotation by an image dorator, for instance. The object wavefront passing through a holographic recording arrangement with a rotating prism (rotating at half the speed of the rotating object) leads to a rotation compensated wavefront to be superimposed on the stationary reference wave, on the recording material. An arrangement is shown in fig. 14. for the vibration analysis of the side-wall of a rotating tyre $(300 \mathrm{rpm})$ using a holographic technique. The road contact is simulated by a toothed wheel. The tyre rotation was counter clockwise. A double pulse holographic arrangement was chosen with a pulse separation of 50 usec. The mode patterns of oscillation near the simulated road contact are clearly visible in fig. 15 . 


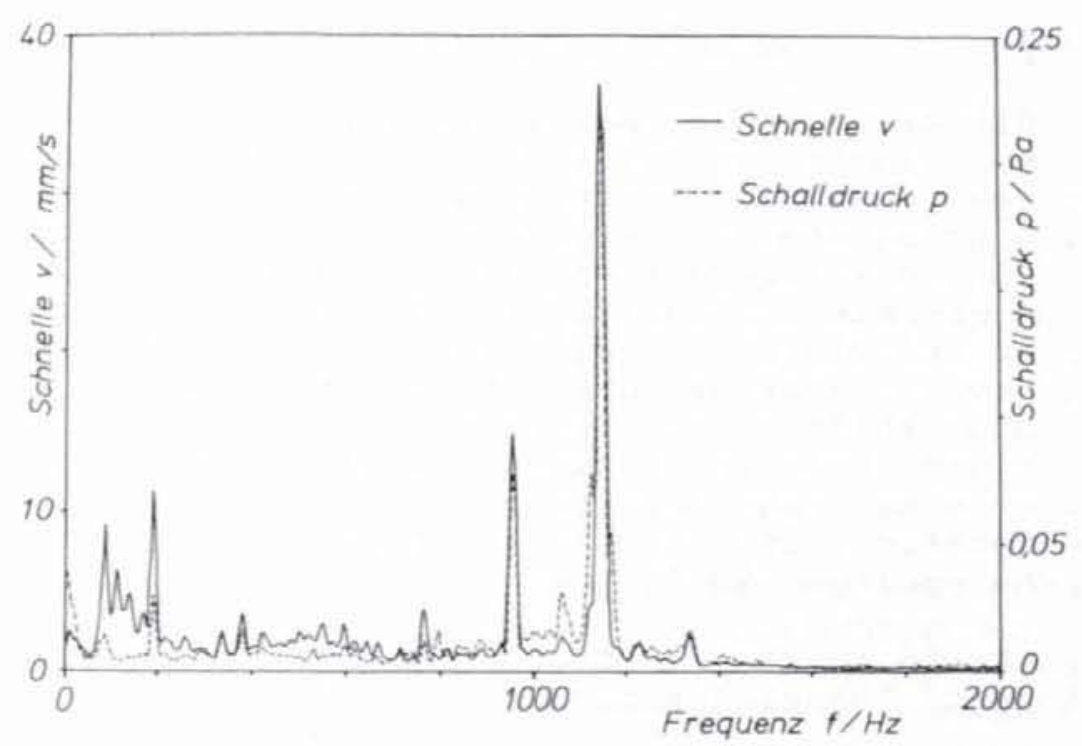

Fig. 13 Noise analysis of the side-wall of a rotating tyre with a microphone and by a Doppler-shift vibrometer for a given point of the rotating tyre. The road contact was simulated by a toothed wheel $(\mathrm{n}=317 \mathrm{rpm})$.

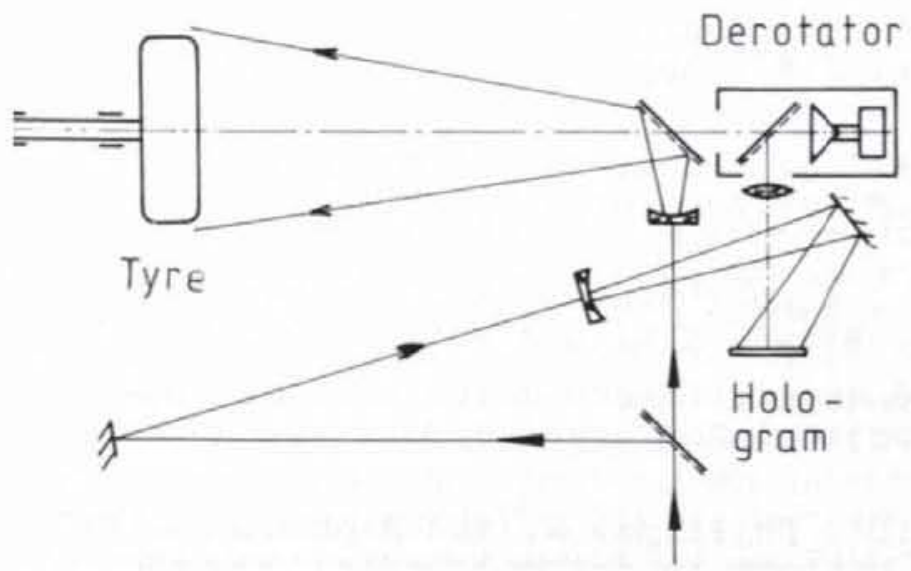

Fig. 14 Arrangement for the vibration analysis of the side-wall of a rotating tyre by holographic interferometry with a double pulse laser and an image derotator

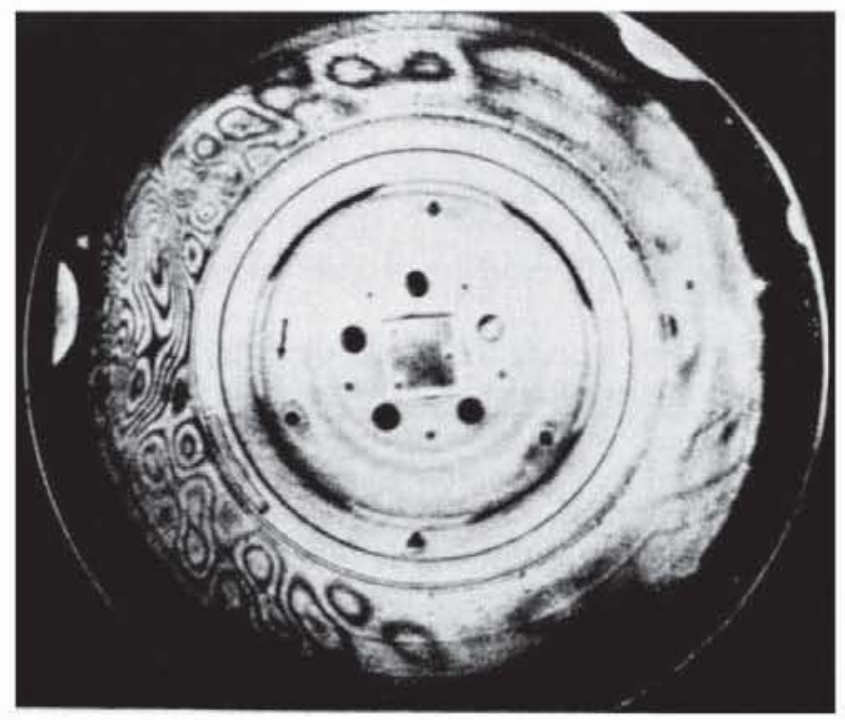

Fig. 15 Mode-patterns of the side-wall of the rotating tyre $(300 \mathrm{rpm})$ with a road contact simulated by a toothed wheel. The fringes were obtained with double pulse holography through an image derotator (pulse separation 50 us) 


\section{Conclusion}

Methods as well as recording materials for real-time measurements were discussed. Electrooptical crystals can be used for nearly realtime contactless measuring techniques. The BSO was found appropriate for real-time holographic interferometry, contour line display and for deformation, displacement and vibration analysis. Furthermore, for real-time speckle photography the BSO was found to be very useful. For noise and vibration analysis of oscillating objects such as rotating car tyres, gearing boxes, turbines blades, a vibrometer based on Doppler shift measuring principles was found to be useful. leading to an amplitude and frequency analysis. For the analyse of rotating objects an image derotator is frequently used for compensation of the rotation. In optical metrology real-time methods will be useful for the engineer in future.

I would like to thank R. Litschel, M. Küchel and J. Klenk for supplying some of the pictures.

\section{REFERENCES}

1. Starkweather, GK., High-speed laser printing systems, in Laser Applications, ed. Goodman, J.W., Monte Ross, Academic Press. (1980)

2. Zernike, F. and Midwinter, J.E., Applied Nonlinear Optics (wiley, New York) 1973

3. Iwasa, s., Optical processing: a near real-time coherent system using two Itek PROM devices, Appl.Opt.Vol 15, pp 1418 - 1424 (1976)

4. Bleha, W.P., Lipton, L.T., Wiener-Avnear, E., Grinberg, J. Reif, P.G., Casasent, D., Brown, H.B. and Markevitch, B.v.

Application of the liquid crystal light valve to real-time optical data processing, Optical Engineering, Vol 17, pp 371 - 84 (1978)

5. Burke, W.J., Staebler, D.L., Phillips, w. and Alphonse, G.A. Volume phase holographic storage in ferroelectric crystals, Optical Engineering, Vol 17, pp 308 - 316 (1978)

6. Huignard, J.P. and Herriau, J.P., Real-time double exposure interferometry with $\mathrm{Bi}_{12} \mathrm{SiO}_{20}$ crystals in transverse electrooptic configuration, Appl. Opt., Vol 16, pp 1807 - 1809 (1977)

7. Marrakchi, A., Huignard, J.P. and Herriau, J.P., Application of phase conjugation in $\mathrm{BI}_{12} \mathrm{~S}_{10} \mathrm{O}_{20}$ crystals to mode pattern visualisation of diffuse vibrating structures, Opt. Communications, Vol 34, pp 15 - 18 (1980)

8. Küchel, M., Tiziani, H.J., Real-time contour holography using BSO crystals, Opt. Communications (inprint)

9. Tiziani, H.J., Leonhardt, K., Klenk, J.

Real-time displacement and tilt analysis by speckle technique using $\mathrm{Bi}_{12} \mathrm{SiO}_{20}$ crystals, Opt. Communications, Vol 34, pp $327^{20} 331$ (1980)

10. Tiziani, H.J., Klenk, J., Vibrationanalysis by speckle techniques in real-time, Appl. Opt., Vol 20, pp 1467 - 1470 (1981) 